\title{
Agreements and controversies of national Guidelines for bronchiolitis: results from an Italian Survey among paediatricians.
}

\author{
Sara Manti ${ }^{1}$, Amelia Licari ${ }^{2}$, Ilaria Brambilla ${ }^{2}$, Carlo Caffarelli ${ }^{3}$, Mauro Calvani ${ }^{4}$, Fabio \\ Cardinale $^{5}$, Giorgio Ciprandi ${ }^{6}$, Claudio Cravidi ${ }^{7}$, Marzia Duse ${ }^{8}$, Alberto Martelli ${ }^{9}$, \\ Domenico Minasi ${ }^{10}$, Michele Miraglia del Giudice ${ }^{11}$, Giovan Battista Pajno ${ }^{12}$, Maria Angela \\ Tosca $^{13}$, Elena Chiappini ${ }^{14}$, and Gian Luigi Marseglia ${ }^{15}$ \\ ${ }^{1}$ University of Catania \\ ${ }^{2}$ Department of Pediatrics, Foundation IRCCS Policlinico San Matteo, University of Pavia \\ ${ }^{3}$ Università di Parma \\ ${ }^{4}$ Ospedale S. Camillo-Forlanini \\ ${ }^{5}$ University of Bari \\ ${ }^{6}$ Casa di Cura Villa Montallegro \\ ${ }^{7}$ ATS Pavia \\ ${ }^{8}$ Policlinico Umberto I, Università Sapienza di Roma \\ ${ }^{9}$ Azienda Ospedaliera G. Salvini, Ospedali di Garbagnate Milanese e Bollate \\ ${ }^{10}$ Grande Ospedale Metropolitano \\ ${ }^{11} 1$ Department of Woman, Child and of General and Specialized Surgery. University of \\ Campania "Luigi Vanvitelli" \\ ${ }^{12}$ Universita degli Studi di Messina \\ ${ }^{13}$ Istituto Giannina Gaslini \\ ${ }^{14}$ University of Florence \\ ${ }^{15}$ Department of Pediatrics, Foundation IRCCS Policlinico San Matteo, University of \\ Pavia, Italy
}

October 1,2020

\begin{abstract}
Introduction. Significant variations in the management of bronchiolitis are often recorded, and, in parallel, to recommend a univocal clinical approach it is not easy and is still questioned. This study is aimed to evaluate the diagnostic and therapeutic management of bronchiolitis in children adopted by Italian paediatricians following the national guidelines. Material and methods. A survey study was designed and carried out by sending an email an open-ended questionnaire developed by an expert panel of the Scientific Board of the Italian Society of Pediatric Allergology and Immunology (SIAIP). Questions were designed according to the national inter-society consensus document on treatment and prevention of bronchiolitis in newborns and infants. Results. Overall, 234 paediatricians were taking part in the study. When diagnosing bronchiolitis, only $44.01 \%$ $(103 / 234)$ of participants were correctly following the national guidelines. All participants (100\%) would perform laboratory tests and/or radiological exams. $44.01 \%$ administered oxygen (O2) when O2 saturation was major than 92\%. About the therapeutic regimen, marked discrepancies between national guidelines and recorded answers were reported. Indications for hospital admission and discharge criteria were in line with the national guidelines. Conclusions. There is a significant practise variation in the management of acute bronchiolitis among Italians physicians. Some wrong attitudes need to be further discouraged, such use of diagnostic procedures and therapeutic approaches. Further research is urgently required to define the
\end{abstract}


best management of patients with bronchiolitis as well as implement strategies to standardize care and improve the quality of care.

\section{Introduction}

Bronchiolitis is the leading cause of hospital admission for respiratory disease among infants younger than one-year-old. Clinically, children with bronchiolitis may present with a wide range of clinical symptoms, from mild to incipient respiratory failure ${ }^{1}$. Diagnosis of bronchiolitis is based on clinical history and a careful physical examination. However, clinical signs, such as rhinorrhea, cough, dyspnea, polypnea, apnea, crackles, wheezing, and feeding difficulties, are not specific and they significantly overlap with symptoms and signs of other diseases ${ }^{2}$. Bronchodilators, corticosteroid, antiviral therapy and methods of oxygen delivery have been suggested as pharmacological interventions in treating acute bronchiolitis; however, the evidence base for their benefit is poor ${ }^{3,4}$. Accordingly, a previous systematic review demonstrated heterogeneous behaviours regarding the adopted diagnostic and therapeutic approaches adopted among several European countries, based on the panel's expertise ${ }^{5}$. Moreover, several aspects are still under debate, including the choice of the best diagnostic and therapeutic management as well as the caregivers' adherence to validated protocols. Therefore, significant variations in the therapeutic management of bronchiolitis between clinicians and hospitals are often recorded, and, in parallel, to recommend a univocal clinical approach, it is difficult and is still questioned ${ }^{3-5}$.

Considering the significant impact of these issues, the Italian Society of Paediatric Allergy and Immunology (SIAIP) conducted a national survey in order to evaluate the behaviours of Italian paediatricians in the diagnostic and therapeutic management of infants and children with acute bronchiolitis and their adherence to the current national recommendations ${ }^{1}$ (Table 1), also aiming to improve the management of bronchiolitis in children and standardize the behaviour of physicians.

\section{Materials and methods}

This study is aimed to evaluate the diagnostic and therapeutic management of bronchiolitis in children adopted by Italian paediatricians in accordance with the national guidelines ${ }^{1}$.

The study was proposed in September 2019 by the Scientific Board of the Italian Society of Pediatric Allergology and Immunology (SIAIP). A specific website was prepared for the study.

The Survey was announced and firstly administered to all the paediatricians attending the "Opinoni a Confronto" Annual Meeting, held in Pavia, Lombardia (Italy) in October 2019. Overall, 100 questionnaires were distributed and full-filled. After that, the study was carried out by sending by email an open-ended questionnaire. Participation in the study was voluntary.

\subsection{Structured questionnaire}

The questionnaire included an explanatory cover letter reporting the aim of the Survey. Experts designed the questions in the field (SM, AL, GLM, EC).

The structured questionnaire was prepared as a specific form to be fulfilled online (closed- and open-ended format questions) on the website. Responses were anonymous, but professional information (university paediatrician, hospital paediatrician, primary care paediatrician, or pediatric resident) was requested. The questionnaire included ten questions in a multiple-choice format. Specifically, the questionnaire contained questions on diagnostic criteria, diagnostic and therapeutic management of bronchiolitis in infants and children younger than 24 months of age. One or more answers for each question were provided.

The survey was administered in Italian and translated into English for publication. The English version of the questionnaire is enclosed in Appendix A.

\subsection{Statistical analysis}


The results were given as absolute numbers and percentages. The percentages of responses to the questions have been calculated on the total of the participants. SPSS software package (SPSS 11.5; Chicago, IL) was used, and $\mathrm{p}<0.05$ was considered as statistically significant.

\section{Results}

The answers to the survey by the participating Italian paediatricians are summarized in figure 1 (a, b, c, d, e, f, g, h, i, l).

\subsection{Participants' characteristics}

Overall, 234 paediatricians (27.7\% of physicians registered to the Congress) returned the questionnaire. The completion rate was $100 \%$.

The average time taken to answer a question was 5 minutes.

The three Italian macro-regions were represented as follow: North 136/234, Centre 55/234, and South $43 / 234$.

The most of participants in the survey were residents in paediatrics $(39.74 \%, 93 / 234)$ followed by hospital paediatricians $(30.34 \%, 71 / 234)$, family paediatricians $(18.8 \%, 44 / 234)$, and university paediatricians $(11.11 \%, 26 / 234)$.

\subsection{Epidemiological findings}

The mean number of patients reporting physician-diagnosed bronchiolitis was $<25(34.18 \%, 80 / 234), 25-50$ $(32.47 \%, 76 / 234),>50(33.33 \%, 78 / 234)$.

\subsection{Diagnostic criteria}

When diagnosing bronchiolitis, $44.01 \%$ (103/234) of participants correctly included the presence of all the following criteria: onset with rhinorrhea and/or upper respiratory tract infections; first episode of respiratory distress associated with crackles and/or wheezing, use of accessory muscles or lower chest wall retractions, low O2 saturation levels, high respiratory rate relative to age, skin colour changes, nasal flaring, fever; exposure to persons presenting with upper respiratory tract viral infections; and presentation during the epidemic season. For 5.5\% (13/234) of participants, diagnostic criteria included rhinorrhea and/or upper respiratory tract infections. For $33.76 \%$ (79/234) of participants, diagnostic criteria included the first episode of respiratory distress associated with crackles and/or wheezing, use of accessory muscles or lower chest wall retractions, low $\mathrm{O} 2$ saturation levels, high respiratory rate relative to age, skin colour changes, nasal flaring, fever. For 6\% (14/234) of participants, diagnostic criteria included exposure to persons presenting with upper respiratory tract viral infections. For $10.68 \%$ (25/234) of participants, diagnostic criteria included clinical presentation during the epidemic season.

\subsection{Approach to the no well-appearing child}

Although neither laboratory tests nor radiological exams are usually indicated for the routine workup of infants with bronchiolitis [1], the proportion of participants who would obtain complete blood count was 43.8\%. $2.37 \%$ would perform blood culture; $85.21 \%$ blood gas analysis, $26.63 \%$ serum electrolytes, $7.69 \%$ glycemia, $46.75 \%$ C-reactive protein $(\mathrm{C}-\mathrm{RP}), 70.41 \%$ polymerase chain reaction (PCR) on the nasal swab, and $13.61 \%$ chest X-ray.

\subsection{Indications for oxygen administration}

$20.08 \%(47 / 234)$ of participants would administer oxygen $\left(\mathrm{O}_{2}\right)$, whether $\mathrm{O}_{2}$ saturation is persistently lower than $90 \%$ and $35.9 \%(84 / 234)$ when $\mathrm{O}_{2}$ saturation ranges between 90 and $92 \%$. Conversely, not following the guideline recommendations [1], 15.81\% (37/234) when $\mathrm{O}_{2}$ saturation ranges between 92 and $94 \%$; and $28.2 \%(66 / 234)$ when $\mathrm{O}_{2}$ saturation is persistently lower than $94 \%$.

3.6 Administration of medications 
Marked discrepancies between national guidelines [1] and recorded answers were reported. The most common treatment administered for bronchiolitis was high flow oxygen therapy $(81.66 \%)$ followed by oxygen therapy with nasal cannula or face mask $(68.05 \%)$, hypertonic solution $(49.70 \%)$, inhaled short-acting beta-agonists (SABA) (39.64\%), inhaled epinephrine (21.30\%), an inhaled corticosteroid (ICS) (17.16\%), systemic CS $(64.52 \%)$, respiratory physiotherapy $(8.88 \%)$, antibiotics $(4.73 \%)$, intravenous SABA $(0.59 \%)$. Among paediatricians using systemic CS, betamethasone was the most common CS administered (64.52\%), followed by methylprednisone $(16.66 \%)$, prednisolone $(7.26 \%)$, beclometasone dipropionate $(4.7 \%)$, and dexamethasone (4.7\%). In line with national guidelines [1], only $16.66 \%$ of the participants did not administer systemic CS.

\subsection{Indications for hospital admission}

The indications for hospital admission included poor feeding or dehydration (98.82\%), comorbidities (98.22\%), need for supplemental oxygen therapy (96.45\%), poor social circumstances $(82.25 \%)$, prematurity $(82.25 \%)$, presence of apnea $(81.66 \%)$, infants less than three months of age $(79.29 \%)$, cyanosis $(78.11 \%)$, tachypnea $(96.44 \%)$, fever $\left(>38^{\circ} \mathrm{C}\right)(44.97 \%)$, uncertain of diagnosis $(29.59 \%)$, unreliable parents $(81.66 \%)$.

\subsection{Discharge criteria}

In line with national guidelines [1], the discharge criteria adopted by interviewed physicians were: improvement in clinical conditions (47.93\%), adequate oral feeding (92.31\%), improved respiratory effort $(92.90 \%)$, improved $\mathrm{O} 2$ saturations $\left(\mathrm{SaO}_{2}>97 \%\right)(52.07 \%)$, improved $\mathrm{O} 2$ saturations $\left(\mathrm{SaO}_{2}>94 \%\right)(37.87 \%)$, improved $\mathrm{O} 2$ saturations $\left(\mathrm{SaO}_{2}>92 \%\right)(5.33 \%)$, carer ability $(78.70 \%)$, adequate social circumstances $(18.34 \%)$, possibility to arrange follow-up (67.46\%).

\section{Discussion}

Significant variations in the management of bronchiolitis are often recorded, and, in parallel, to recommend a univocal clinical approach it is difficult and is still questioned. This study aimed to evaluate the diagnostic and therapeutic management of bronchiolitis in children adopted by Italian paediatricians in accordance with the national guidelines. Our survey showed a significant practice variation in the management of acute bronchiolitis among Italians physicians.

Nationally developed evidence-based guidelines are required to reduce practice variation, minimize nonevidence-based practices, and promote cost-effective standardization of care $^{6}$. Nevertheless, a significant practice variation persists, and it has been previously reported that several guideline recommendations have been poorly incorporated into the real clinical settings after their development and dissemination ${ }^{7,8}$. Accordingly, several international studies have detected considerable variation in the management of acute bronchiolitis ${ }^{9}$. A significant practice variation in the monitoring, treatment, and discharge of the children hospitalized with bronchiolitis among physicians across Italian paediatric hospitals has also been founding ${ }^{5}$. Therefore, given the high risk of inappropriate and unnecessary medicalization and hospitalizations of patients with bronchiolitis and, consequently, the bronchiolitis-related high-financial burden, it is important to examine the diagnostic and therapeutic interventions administered in infants presenting with bronchiolitis. In this regard, the SIAIP conducted a national survey to evaluate the behaviours of Italian paediatricians in the diagnostic and therapeutic management of infants and children with acute bronchiolitis and their adherence to the current national recommendations. To the best of our knowledge, a similar investigation had not yet been conducted in Italy, making this study essential for understanding the Italian management of patients suffering from bronchiolitis. This information would also clarify the use of evidence-based supportive therapies as compared with those that are not evidence-based, aiming to improve the management of bronchiolitis in children and standardize the behaviour of physicians.

The diagnosis of bronchiolitis is based on clinical history and physical examination ${ }^{1}$. In accordance to the national guidelines ${ }^{1},<50 \%$ participants agreed on the diagnostic criteria, and the most common criteria for bronchiolitis diagnosis were onset with rhinorrhea and/or upper respiratory tract infections; the first episode of respiratory distress associated with: crackles and/or wheezing, use of accessory muscles or lower chest wall retractions, low $\mathrm{O} 2$ saturation levels, high respiratory rate relative to age, skin colour changes, nasal flaring, 
fever; exposure to persons presenting with upper respiratory tract viral infections; and presentation during the epidemic season. Marked discrepancies could be observed between the remaining respondents as well as inappropriateness in the answer.

National guidelines include as criteria for hospital admission the following: O2 saturation persistently lower than $90-92 \%$, the entity of respiratory distress, presence of apnea, dehydration, and moderate to severe bronchiolitis ${ }^{1}$. Other important factors should be taken into consideration are prematurity (gestational age $<37$ weeks or birth age $<6-12$ weeks), responsivity and alertness, poor hydration and feeding (defined as less than $50 \%$ of usual fluid intake in preceding $24 \mathrm{~h}$ ), social and environmental factors, and presence of pre-existing risk factors ${ }^{1}$.

The decision to hospitalize a child affected by bronchiolitis is a complex process impacted by the course of the illness and other clinical, socio-cultural and geographic factors as well as by the possibility to access to the follow-up ${ }^{1,10}$. Moreover, the local culture of care can influence the decision-making process, irrespective of disease severity. Thus, although inpatient observation is recommended for selected infants affected by bronchiolitis, physicians are more likely to hospitalize infants with milder bronchiolitis, taking improper advantage of the use of inpatient resources. On the other hand, the variability in admission criteria among guidelines may alter unavoidably the hospitalization criterion. In this regard, it must be considered that some institutions have protocols that require the ordering of diagnostic tests (e.g., complete blood count, chest Xray) on admission. However, a priori reasons to support this clinical practice are not currently supported by evidence-based medicine $(\mathrm{EBM})^{11}$. Moreover, this practice could lead both to perform costly and unnecessary testing/procedure cascade as well as inappropriate outpatient/inpatient antibiotic prescriptions.

The administration of non-recommended interventions in our study occurred at a moderate-to-high rate, as follows: inhaled epinephrine (21.30\%), ICS (17.16\%), systemic CS (64.52\%), respiratory physiotherapy $(8.88 \%)$, antibiotics $(4.73 \%)$, intravenous SABA $(0.59 \%)$. The current literature does not recommend the diagnostic testing for children with bronchiolitis, except for conducting epidemiological studies. Moreover, very few data support the impact of testing on patient outcomes and quality of care, and they do not provide clear indications for such testing or the impact of testing on relevant patient outcomes ${ }^{1,5,12}$. Moreover, the overuse of instrumental diagnostic tests does not comply with patient safety and, also, weighs on the healthcare economy.

Elsewhere, the guidelines developed by a panel of experts aim to minimize overtreatment by recommending against the use of noneffective interventions ${ }^{13-19}$. Nevertheless, authors of inpatient bronchiolitis studies published after the 2006 American Academy Pediatrics (AAP) bronchiolitis guideline have found disappointingly high use of the non recommended resources ${ }^{20,21}$. We found a surprising number of children were treated with medications. Following the Italian guidelines ${ }^{1}$, supplemental oxygen $\left(\mathrm{O}_{2}\right)$ should be administered if $\mathrm{O}_{2}$ saturation levels are persistently below $90-92 \%$ at ambient air ${ }^{1,22}$.

A recent trial has suggested that outcomes may not differ significantly when an $\mathrm{O}_{2}$ saturation target of [?] $90 \%$ is used ${ }^{23}$. Whether an increased respiratory effort is occurring, high-flow oxygen therapy with humidified and heated oxygen (high-flow nasal cannula, HFNC) should be considered. However, different $\mathrm{O}_{2}$ saturation thresholds are recommended for hospital management and for starting $\mathrm{O}_{2}$ therapy. Thus, it is reasonable to hypothesize that both the difference in the evidence provided by the literature as well as context-specific factors may be key reasons for the differences in clinical practice. In children with bronchiolitis who cannot maintain oral hydration intravenous or nasogastric fluids may be considered ${ }^{1,22}$. There is sparse evidence supporting the routine use of hypertonic solution, nebulized adrenaline, salbutamol, respiratory physiotherapy, systemic or inhaled or systemic CS, and antibiotics ${ }^{1,22}$. However, authors support the use of hypertonic saline to decrease airway oedema and improve mucociliary clearance in infants ${ }^{1,22}$. Unlikely to other respiratory diseases in which CS show a beneficial effect, their use does not improve the short- and long-term prognosis in infants with bronchiolitis ${ }^{1,22}$.

Similarly, the association of systemic CS with epinephrine or SABA does not produce a significant benefit ${ }^{1,22}$. The administration of bronchodilators in managing bronchiolitis is still contentious and, although evidence 
is against their routine use, several guidelines still recommended a trial of bronchodilators in treating infants with acute bronchiolitis ${ }^{5}$. Despite the absence of evidence suggesting a benefit of the use of antibiotic in acute bronchiolitis, their prescription in primary care settings is commonly reported ${ }^{1,22}$. Evidence supporting the use of nebulized adrenaline, antivirals, antileukotrienes, and chest physiotherapy (vibration and percussion techniques) during the acute phase of bronchiolitis is insufficient ${ }^{1}$.

Surprisingly, regarding the discharge criteria, the healthcare professional behaviour was in line with the national guidelines ${ }^{1}$. An appropriate discharge requires evaluation of multiple factors including medical (clinical conditions, adequate feeding, improved respiratory effort), psychosocial (carer ability), logistic (possibility to arrange follow-up), and economic (adequate social circumstances) considerations. Discharge planning must involve both the health care team and patient/family caregivers to develop a patient-centred plan ensuring that the patient is safely discharged home and minimizing the risk of adverse events and/or unplanned readmissions ${ }^{22}$.

Except for the data on the discharge criteria, we formulated different hypotheses to explain the striking differences between the findings of our study and the guideline-related recommendations of nonintervention. Acute bronchiolitis significantly causes pressures in health service and throughout the region. Probably, the parental expectation "to do something" might be in part responsible for these results, also increasing the local practice differences. In a previous study, authors reported that bronchiolitis hospitalization caused significant emotional, physical and organizational consequences both on parents and siblings persisting up to 3 months after hospital discharge ${ }^{25}$. Moreover, it is possible that primary care paediatricians, who may have limited experience with managing acute bronchiolitis in advanced stage of disease, are influenced wrongly by the medical literature showing benefit of SABA and/or ICS in other respiratory diseases ${ }^{22,26}$. Furthermore, there is evidence that exposure to a few cases of patients with bronchiolitis is associated with the increased use of investigations and medications ${ }^{27}$. Lastly, the difficulty and/or the impossibility to arrange follow-up care can affect disease management.

\subsection{Limits of the study}

The survey design generates findings entirely based on recall over a previous calendar year. However, given the fact there has been no national or local implementation of a standardized bronchiolitis guideline, we can exclude with reasonable certainty that a significant change in clinical practice has occurred since the time our data were recorded. Furthermore, what physicians report and what they do in real clinical practice may often vary. Additionally, data were only collected from 234 paediatricians, but this denominator can not be definitively representative of all nation. Moreover, small sample sizes in some sites have limited our ability to investigate site-to-site variation. On the other hand, the solid response rate and the broad representation of sites across the whole country lend weight and credibility to our results.

\section{Conclusions}

There is a significant practice variation in the monitoring, treatment, and discharge of children suffering from acute bronchiolitis among Italians physicians. Future research is urgently required to define the best management of patients with bronchiolitis, optimize the use of therapeutic resources as well as implement strategies to standardize care and improve the quality of care.

\section{References}

1. Baraldi E, Lanari M, Manzoni P, Rossi GA, Vandini S, Rimini A, Romagnoli C, Colonna P, Biondi A, Biban P, et al. Inter-society consensus document on treatment and prevention of bronchiolitis in newborns and infants. Ital J Pediatr. 2014;40:65

2. Bronchiolitis Guideline Team, Cincinnati Children's Hospital Medical Center. Evidence-Based Clinical Practice Guideline for Medical Management of Bronchiolitis in Infants 1 Year of Age or Less Presenting with a First Time Episode. Cincinnati: Cincinnati Children's Hospital Medical Center; 2010

3. Management of Bronchiolitis in Infants. The Hospital for Sick Children, Clinical Practice Guideline. 2011 
4. Bialy L, Foisy M, Smith M, Fernandes RM. The Cochrane Library and the treatment of bronchiolitis in children: an overview of reviews. vid Based Child Health 2011; 6:258-75

5. Kirolos A, Manti S, Blacow R, Tse G, Wilson T, Lister M, Cunningham S, Campbell A, Nair H, Reeves RM, et al. A Systematic Review of Clinical Practice Guidelines for the Diagnosis and Management of Bronchiolitis. J Infect Dis. 2019;jiz240

6. Perlstein PH, Kotagal UR, Bolling C, Steele R, Schoettker PJ, Atherton HD, Farrell MK. Evaluation of an evidence-based guideline for bronchiolitis. Pediatrics 1999;104 (6):1334-41

7. Meehan WPIII, Fleegler E, Bachur RG. Adherence to Guidelines for managing the well-appearing febrile infant: assessment using a case-based, interactive survey. Pediatr Emerg Care. 2010;13:875-880

8. Grol R. Successes and failures in the implementation of evidence-based guidelines for clinical practice. Med Care. 2001;13:46-54

9. Schuh S, Babl FE, Dalziel SR, Freedman SB, Macias CG, Stephens D, Steele DW, Fernandes RM, Zemek R, Plint AC,et al. Practice Variation in Acute Bronchiolitis: A Pediatric Emergency Research Networks Study. Pediatrics. 2017;140(6):e20170842

10. Zamor R, Byczkowski T, Zhang Y, Vaughn L, Mahabee-Gittens EM. Language Barriers and the Management of Bronchiolitis in a Pediatric Emergency Department. Acad Pediatr. 2020;20(3):356-363.

11. Tarini BA, Garrison MM, Christakis DA. Instit utional variation in ordering complete blood counts for children hospitalized with bronchiolitis. J Hosp Med. 2007;2(2):69-73

12. Bordley WC, Viswanathan M, King VJ, Sutton SF, Jackman AM, Sterling L, Lohr KN. Diagnosis and testing in bronchiolitis: a systematic review. Arch Pediatr Adolesc Med. 2004;158(2):119-126.

13. American Academy of Pediatrics Subcommittee on Diagnosis and Management of Bronchiolitis. Diagnosis and management of bronchiolitis. Pediatrics.2006;118(4):1774-1793

14. Ralston SL, Lieberthal AS, Meissner HC, Alverson BK, Baley JE, Gadomski AM, Johnson DW, Light MJ, Maraqa NF, Mendonca EA, et al. American Academy of Pediatrics. Clinical practice guideline: the diagnosis, management, and prevention of bronchiolitis. Pediatrics. 2014;134(5).

15. Friedman JN, Rieder MJ, Walton JM; Canadian Paediatric Society, Acute Care Committee, Drug Therapy and Hazardous Substances Committee. Bronchiolitis: Recommendations for diagnosis, monitoring and management of children one to 24 months of age. Paediatr Child Health. 2014;19(9):485-498

16. Scottish Intercollegiate Guidelines Network. Bronchiolitis in Children: A National Clinical Guideline. Edinburgh, UK: Scottish Intercollegiate Guideline Network; 2006. Guideline No. 91

17. Turner T, Wilkinson F, Harris C, Mazza D; Health for Kids Guideline Development Group. Evidence based guideline for the management of bronchiolitis. Aust Fam Physician. 2008;37(6 spec no):6-13

18. Barben J, Kuehni CE, Trachsel D, Hammer J; Swiss Paediatric Respiratory Research Group. Management of acute bronchiolitis: can evidence based guidelines alter clinical practice? Thorax. 2008;63(12):1103-1109

19. National Institute for Health and Care Excellence. Bronchiolitis in children: diagnosis and management. 2015. Available at: https:// www. nice. org. uk/guidance/ ng9. Accessed June 24, 2016

20. Cheung CR, Smith H, Thurland K, Duncan H, Semple MG. Population variation in admission rates and duration of inpatient stay for bronchiolitis in England. Arch Dis Child. 2013;98(1):57-59

21. Working Group of the Clinical Practice Guideline on Acute Bronchiolitis; Sant Joan de Deu Foundation Fundacio Sant Joan de Deu, coordinator; Clinical Practice Guideline on Acute Bronchiolitis; Quality Plan for the Spanish National Healthcare System of the Spanish Ministry for Health and Social Policy; Catalan Agency for Health Technology Assessment. Clinical Practice Guideline on Acute Bronchiolitis. 2010. Available at: http://www. guiasalud. es/ GPC/ GPC_ 475_Bronchiolitis_ AIAQS_compl_en. pdf

22. Cutrera R, Baraldi E, Indinnimeo L, Miraglia Del Giudice M, Piacentini G, Scaglione F, Ullmann N, Moschino L, Galdo F, Duse M. Management of acute respiratory diseases in the pediatric population: the role of oral corticosteroids. Ital J Pediatr. 2017;43(1):31.

23. Cunningham S, Rodriguez A, Adams T, Boyd KA, Butcher I, Enderby B, MacLean M, McCormick J, Paton JY, Wee F, et al. Oxygen saturation targets in infants with bronchiolitis (BIDS): a double-blind, randomised, equivalence trial. Lancet. 2015;386(9998):1041-1048 
24. Alper E, O'Malley T, Greenwald J. Hospital discharge and readmission. UpToDate- 2020, June 16th. Last access on August 9th, 2020. Available at: https://www.uptodate.com/contents/hospitaldischarge-and-readmission.

25. Lapillonne A, Regnault A, Gournay V, Gouyon JB, Gilet H, Anghelescu D, Miloradovich T, Arnould B, Moriette G. Impact on parents of bronchiolitis hospitalization of full-term, preterm and congenital heart disease infants. BMC Pediatr. 2012;12:171.

26. Christakis DA, Cowan CA, Garrison MM, Molteni R, Marcuse E, Zerr DM. Variation in inpatient diagnostic testing and management of bronchiolitis. Pediatrics 2005;115(4):878-84

27. Van Cleve WC, Christakis DA. Unnecessary care for bronchiolitis decreases with increasing inpatient prevalence of bronchiolitis. Pediatrics 2011;128(5):e1106-12.

\section{Table}

Table 1. The basic diagnostic and therapeutic management of bronchiolitis in accordance with the national recommendations [1].

\section{Appendix}

Appendix A. English version of the questionnaire administered.

\section{Figure}

Fig. 1 (a, b, $\mathbf{c}, \mathbf{d}, \mathbf{e}, \mathbf{f}, \mathbf{g}, \mathbf{h}, \mathbf{i}, \mathbf{l})$. Answers provided by the participating Italian pediatricians to the survey. (a, b) Participants' characteristic (data are expressed in absolute value). (c) Mean number of patients reporting physician-diagnosed bronchiolitis (data are expressed in absolute value). d) Diagnostic criteria: A: rhinorrhea and/or upper respiratory tract infections; B: first episode of respiratory distress associated with crackles and/or wheezing, use of accessory muscles or lower chest wall retractions, low O2 saturation levels, high respiratory rate relative to age, skin colour changes, nasal flaring, fever; exposure to persons presenting with upper respiratory tract viral infections; and presentation during the epidemic season; C: persons presenting with upper respiratory tract viral infections; D: clinical presentation during the epidemic season; E: all the previous criteria (data are expressed in percentage). e) Approach to the no well-appearing child: A: complete blood count, B: blood culture; C: blood gas analysis; D: serum electrolytes; E: glycemia; F: C-reactive protein $(\mathrm{C}-\mathrm{RP})$; G: polymerase chain reaction (PCR) on the nasal swab; H: chest X-ray (data are expressed in percentage). f) Indications for oxygen administration (data are expressed in percentage). g) Administration of medications: A: high flow oxygen therapy; B: oxygen therapy with nasal cannula or face mask; C: hypertonic solution; D: inhaled short-acting beta-agonists; E: inhaled epinephrine; F: inhaled corticosteroid; G: systemic corticosteroid; H: respiratory physiotherapy; I: antibiotics; L: intravenous short-acting beta-agonists; M: antivirals; N: antileukotrienes; O: systemic epinephrine (data are expressed in percentage). h) The most common corticosteroids administered: A: betamethasone, B: methylprednisone, C: prednisolone, D: beclometasone dipropionate, E: dexamethasone; F: none (data are expressed in percentage). i) Indications for hospital admission: A: infants less than 3 months of age; B: prematurity; C: respiratory rate $>30$ breaths/minute; D: respiratory rate $>50$ breaths/minute; E: respiratory rate $>60$ breaths/minute; F: poor feeding or dehydration; G: cyanosis; H: apnea; I: comorbidities; L: poor social circumstances; M: uncertain of diagnosis; N: need for supplemental oxygen therapy; O: fever; P: unreliable parents (data are expressed in percentage). l) Discharge criteria: A: $\mathrm{SaO} 2>97 \%$, B: $\mathrm{SaO} 2>94 \%$; C: $\mathrm{SaO} 2>92 \%$; D: improved respiratory effort; E: adequate oral feeding; F: adequate social circumstances; G: carer ability; H: possibility to arrange follow-up; I: improvement in clinical conditions (data are expressed in percentage). 

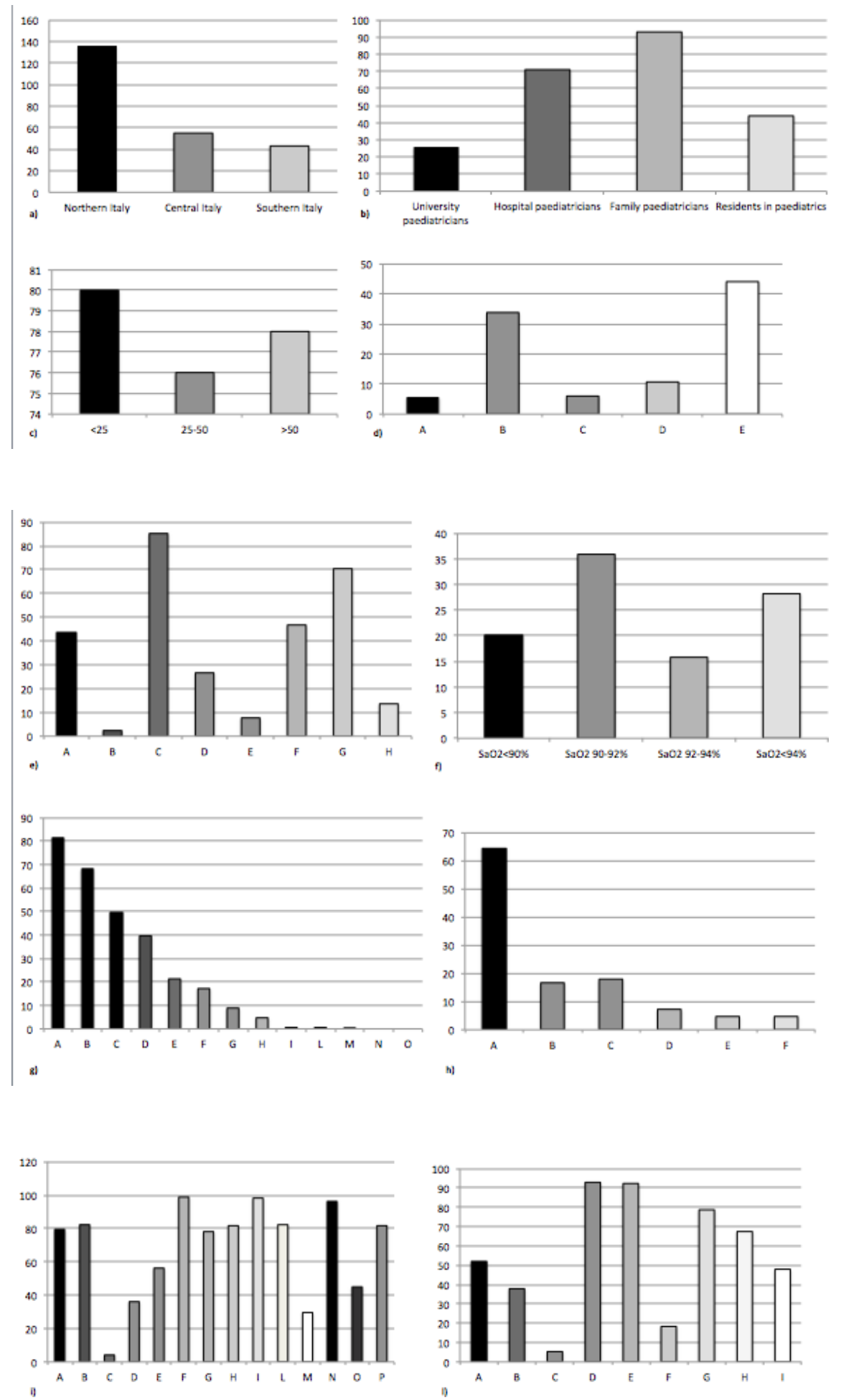

\section{Hosted file}

Table.pdf available at https://authorea.com/users/340024/articles/484289-agreements-andcontroversies-of-national-guidelines-for-bronchiolitis-results-from-an-italian-survey- 
among-paediatricians 